\title{
A Scaling Method for Video Images Based on Nonlinear Curve
}

\author{
Ma Shexiang ${ }^{1,}$, Wang Sibo $^{1, b}$ \\ ${ }^{1}$ School of Computer and Communication Engineering, Tianjin University of Technology, Tianjin \\ 300384, China \\ amasx_tjut@126.com, ${ }^{\mathrm{b}}$ sieber501@163.com
}

Keywords: nonlinear scaling, scaling curve, elliptic curve function, nonlinear scaling factor

\begin{abstract}
When Digital Video image transformation from general screen (i.e., 4:3, etc.) to widescreen (i.e., 16:9, etc.), the source image aspect ratio will change, image resolution adjustment and format conversion is difficult. In this paper, a method of nonlinear scaling is proposed, aim to overcome the image edge distortion problem caused by conventional transform methods. The proposed method is to transform based on nonlinear scaling curve in the horizontal direction, it uses elliptic curve scaling function on both sides of the image and also add appropriate nonlinear scaling factor in central area, achieving the overall image of the nonlinear scaling factor change is smooth, to reduce the overall image distortion. The simulation result shows that the distortion of the scaled image is acceptable, the proposed method is more efficient than conventional methods.
\end{abstract}

\section{Introduction}

In the television and display devices, the common video image display mode are 4:3 and 16:9. When transformation among different display modes or formats of the image, the resolution transformation caused by the image aspect ratio change [1] will lead to image distortion. To transform the original 4:3 video image into 16:9 image, the authors in [2,3] compared with the three methods: letter-box mode [4], crop mode [5] and expand mode, the image scaling effects are not satisfactory. A nonlinear scaling method is proposed in [6,7], the authors keep linear scaling in the middle of the original image, and nonlinear change on both sides, this method not only ensure no distortion in the middle part and guarantee the integrity of the image. However, there are severe deformation on both sides of the image, in sharp contrast to the central part, the overall image distortion is obvious. In this paper, we propose a new scaling method for Video Images based on nonlinear curve.The method utilizes a nonlinear curve for image scaling, and add a suitable smaller scale factor on the minimum value in center of the curve, to achieve the overall image more smooth change and smaller image deformation. The proposed method can realize image scaling, and it is more efficient than conventional methods.

\section{Literature Review}

Shi Zaifeng et al in [7] proposed the nonlinear scaling method for video images, the principle of this method as shown in Fig.1.

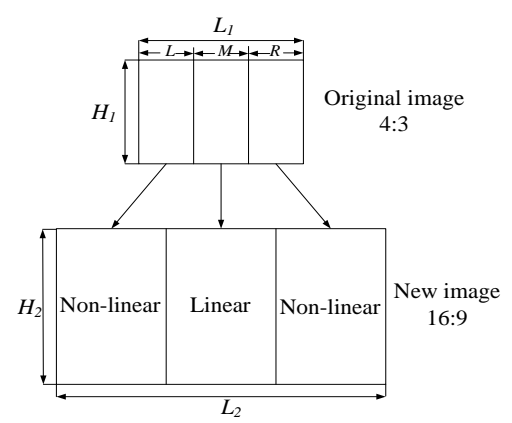

Fig.1 conventional nonlinear scaling method 
$M$ is linear area in original video images, $L$ and $R$ are equal and both are nonlinear scaling. In the original image, horizontal resolution is $L_{1}$ and vertical resolution is $H_{1}$. In the new image, horizontal resolution is $L_{2}$ and vertical resolution is $H_{2}$. Let $r=H_{2} / H_{1}=2.25, r$ is the magnification ratio for vertical direction and the overall linear area.

The interpolate points in $M$ are evenly distributed and interpolation points distance is $1 / r$, however, those in $L$ and $R$ are unevenly distributed. In horizontal direction, the interpolation principle of nonlinear scaling area use the arithmetic sequence function and sine curve. The effect between the two nonlinear algorithms using the arithmetic curve and the sine curve has no significant difference, but the amount of calculation and implementation difficulty of sine curve are greater than that of arithmetic curve. Considering the resource consumption of hardware implementation, generally, using arithmetic curve as a nonlinear interpolation curve to scale image.

\section{Proposed Method}

In this paper, the interpolation principle of the proposed method is the original image pixels nonlinear mapping to the target image pixels position, then interpolate the target image.

\section{A. Proposed interpolation method}

In the mapping from the original image to the target image, the original image is magnified $r$ times in the vertical direction, then nonlinear scaling the image in the horizontal direction. After the nonlinear change, using appropriate interpolation algorithm to interpolate in the blank pixels position, the interpolation process is shown in Fig.2.

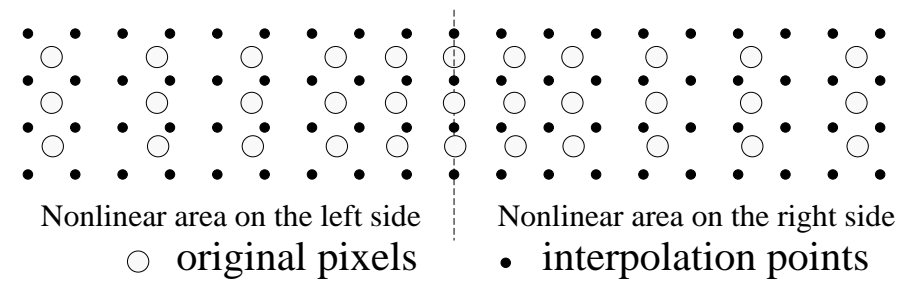

Fig.2 Proposed interpolation method

As shown in Figure.2, the distance between the original pixels nonlinear enlarged. Proposed method adopts bilinear interpolation algorithm.

\section{B. The design of nonlinear scaling curve}

Translate the above-described method described the image scaling effect by interpolation points density into nonlinear scaling method using nonlinear scaling factor. Therefore, introducing nonlinear scaling factor which is the ratio of new image pixel pitch and the original image pixel pitch in the horizontal direction. Using the nonlinear scaling factor principle, the above-described method's linear area scaling is $r$, and the nonlinear scaling factor in the nonlinear area can be obtained by the arithmetic function in the horizontal direction. In this paper, in order to realize the smooth transition, we choose the concave curve to be nonlinear scaling curve. When using the concave curve, the scaling factor at the edges of nonlinear area change significantly, we need add an appropriate amplification factor on the center area to reduce the contrast of both sides and the central area and the image distortion. When selecting a concave curve, we compared sine curve, quadratic curve and elliptic curve. The elliptic function curve has the maximum concave degree, and the overall change is smooth, this can guarantee the center area display effect and reduce the distortion. 
Firstly, proposed method will expand $r$ multiples in vertical direction of the original image, then nonlinear scaling the image in the horizontal direction according to the elliptic curve method in Fig. 3

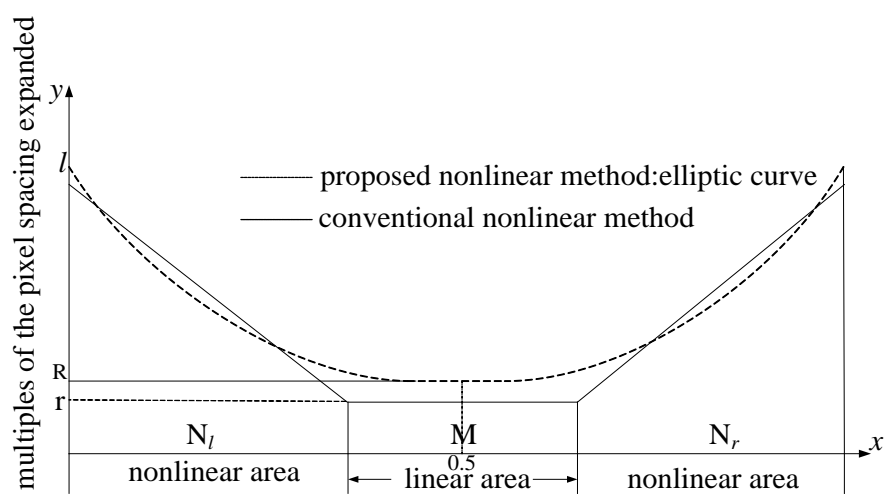

Fig. 3 Comparison of proposed method with conventional nonlinear method

Define $R$ as the minimum value of the elliptic curve, $R=r+\delta, \delta$ is a small scale factor that can be taken reasonable value by simulation experiment. $N_{l}$ and $N_{r}$ represent ratio of the nonlinear scaling area on both sides of the original image for the entire image [7], $N_{l}=N_{r}=(1-M) / 2$, the total scaling of the horizontal direction is $r^{\prime}=L_{2} / L_{1}=3$. The elliptic curve function can be expressed according to the parameters in Fig. 3, the nonlinear scaling curve function is:

$$
y=l-(l-R) \sqrt{1-\frac{(x-0.5)^{2}}{(0.5)^{2}}}
$$

Multiple relationship equation can be expressed as:

$$
\int_{0}^{1} y d x=r^{\prime}
$$

After simplifying equation, we can obtain the relationship equation of maximum scaling $l$ and the minimum magnification $R$ :

$$
(1-\pi / 4) l+(\pi / 4) R=3
$$

\section{Simulation Results}

In the simulations, conventional nonlinear method[7] $M=0.4, r=2.25$, proposed method $M=0$, $R=2.5$. Compare the effects of proposed nonlinear scaling method with expand mode, conventional nonlinear method [7], the results of simulation experiment are shown in Fig.4, Fig.5.

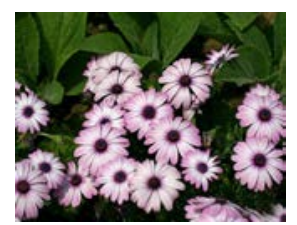

(a) original image

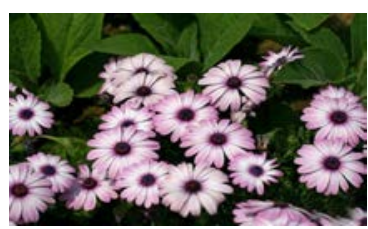

(b) expand mode

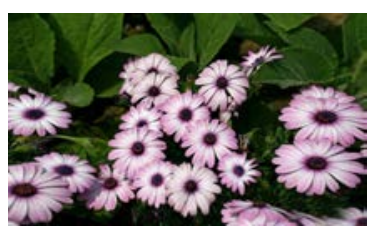

(c) conventional method[7]

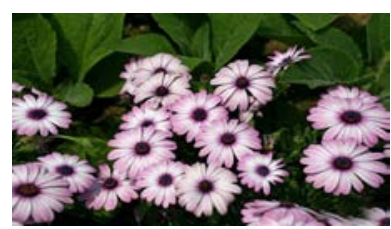

(d) proposed method

Fig 4 Multiple interest targets in the image

As shown in the Fig.4, the original image has multiple interest targets. The image distortion is serious in Fig.4(b). Fig.4(c) is stretched seriously at the edges of the image. Fig.4(d) is the nonlinear amplification effect by the proposed methed, it has a better scaling effect than other methods. 


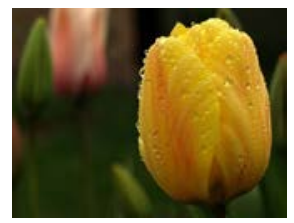

(a) original image

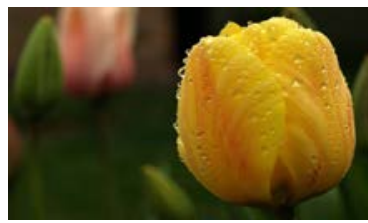

(b) expand mode

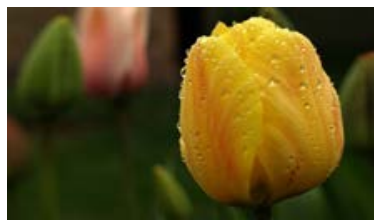

(c) conventional method[7]

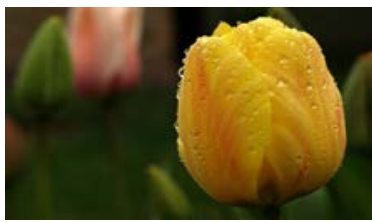

(d) proposed method

Fig.5 Interest targets on both sides of the image

Fig. 5 shows comparison of image scaling methods when the interest targets on both sides of the image. In the Fig.5(b), the flower has significant deformation. The flower and bud are obvious deformation on both sides of Fig.5 (c). Compared Fig. 5 (d) with Fig.5 (b) and Fig.5(c), Fig. 5 (d) has a good amplification effect by the proposed method.

\section{Conclusion}

In this paper, we propose a new nonlinear scaling method baesd on elliptic curve, which can acheve scaling images among the images of different aspect ratios. After the original image pixel are mapped to a new target image pixel position, using bilinear interpolation algorithm. The proposed method makes the contrast of the nonlinear scaling area decrease, and reduce the distortion degree of the overall image, the scaling effect is superior to other methods and the algorithm complexity is lower.

\section{Acknowledgements}

Project supported by the National Nature Science Foundation of China (No.61371108) and High School Science and Technology Fund Planning Project of Tianjin (No.20140706, No.20140707).

\section{References}

[1] Hans Hoffmann, Takebumi Itagaki, A Novel Method for Subjective Picture Quality Assessment and Further Studies of HDTV Formats. IEEE Transactions on Broadcasting, 2008, 54(1): 1-1.

[2] Weijun Ren, Hongdong Chu, Yuyao He. Nonlinear Scaling Algorithm of Video Image Aspect Ratio Based on Changing Zone. Video Engneering, 2008, 32 (311): 41-42. “ In Chinese“

[3] Zhongliang Deng, Yandong Guo, Xiaodong Gu. A comparative review of aspect ratio conversion methods, Proceedings of International Conference on Multimedia and Ubiquitous Engineering, Busan, 2008: 114 117.

[4] Jechang Jeong, Byeungwoo Jeon. A Multiplierless Letter-Box Converter for Displaying 16:9 Images on a 4:3 Screen. IEEE Transactions on circuits and systems for video technology, 1995, 5(4): 363-366.

[5] Hsia Shih-Chang, Liu Bin-Da. A parallel video converter for displaying 4:3 images on 16:9 HDTV receivers. IEEE Transactions on Circuits and Systems for Video Technology, 1996, 6(6): 695-699.

[6] Hsia Shih-Chang, Liu Bin-Da, Yang Jar-Ferr, et al. An NTSC to HDTV video conversion system by using the block processing concept. Proceedings of IEEE Transactions on Consumer Electronics. Rosemont, USA, 1994: 216-224.

[7] Zaifeng Shi, Xia Xiao, Kai Shao, et al. A Novel Nonlinear Scaling Method for Video Images. Proceedings of International Conference on Computer Science and Software Engineering. Wuhan, China, 2008: 357-360. 